\title{
Sensitivity of the performance of a conceptual rainfall- runoff model to the temporal sampling of calibration data
}

\author{
Satish Bastola and Conor Murphy
}

\begin{abstract}
The effect of the time step of calibration data on the performance of a hydrological model is examined through a numerical experiment where HYMOD, a rainfall-runoff model, is calibrated with data of varying temporal resolution. A simple scaling relationship between the parameters of the model and modelling time step is derived which enables information from daily hydrological records to be used in modelling at time steps much shorter than daily. Model parameters were found to respond differently depending upon the degree of aggregation of calibration data. A loss in performance, especially in terms of the Nash-Sutcliffe measure, is evident when behavioural simulators derived with one modelling time step are used for simulation at another time step. The loss in performance is greater when parameters derived from a longer time step were used for simulating flow with a shorter time step. The application of a simple scaling relationship derived from a multi-time step model calibration significantly decreased the loss in model performance. Such an approach may offer the prospect of conducting higher temporal resolution flood frequency analysis when finer scale data for model calibration are not available or limited.
\end{abstract}

Satish Bastola (corresponding author) COAPS,

Florida State University,

Tallahassee,

FL 32306,

USA

E-mail: sbastola@coaps.fsu.edu

Conor Murphy

Irish Climate Analysis and Research Units (ICARUS), Department of Geography,

National University of Ireland Maynooth,

Maynooth,

County Kildare,

Ireland

Key words | multi-objective calibration, rainfall-runoff model, temporal aggregation

\section{INTRODUCTION}

Conceptual hydrological models are widely used for informing the practical management of water resources. Such uses range from real time flood forecasting to the simulation of future climate change impacts (Bastola et al. 20II). In applying models the appropriate simulation time step should reflect the timing and scale of key processes of interest. The aggregation of model input in time affects the ability of a model to capture small-scale processes, thereby affecting the identifiability of related model parameters. Poor identifiability has notable implications for prediction uncertainty, understanding hydrological processes and in extending the use of hydrological models as a tool for prediction in ungauged basins.

As a key input, the temporal and spatial pattern of rainfall plays an important role in determining the hydrological response of river basins. Therefore, the influence of these rainfall characteristics in simulating hydrological response has been widely investigated (e.g., Krajewski et al. I99I; doi: $10.2166 /$ nh.2012.061
Finnerty et al. 1997; Ishidaira et al. 2003; Littlewood \& Croke 2008; Wang et al. 2009). Ostrowski et al. (2010) briefly summarize past discussions (predominantly from the early 1980s) on the dependency of derived model parameters on the rainfall time step used for calibration. More recently, a number of authors have recognized the need to identify the scale dependencies of critical hydrologic parameters (e.g., Littlewood \& Croke 2008; Wang et al. 2009).

Runoff scaling experiments conducted by Finnerty et al. (I997) show that the output from a lumped hydrological model was sensitive to the spatial and temporal averaging of rainfall inputs. They also presented a preliminary approach for adjusting model parameters to account for spatial and temporal variation in rainfall input. Krajewski et al. (I99I) reported the greater influence of the temporal resolution of rainfall inputs over spatial variability on model output. More recently, the work of Littlewood \& Croke (2008), Wang et al. (2009) and Ostrowski et al. (20I0) 
has explored the time scale dependencies of hydrological model parameters. These studies show that the parameters describing different components of runoff response, their identifiability and derived values, depend upon the temporal sampling of the calibration data.

In particular, the parameters describing slow flow processes reveal a low sensitivity, usually remaining constant over the range of temporal scales, while parameters describing quick flow components showed high sensitivity and dependence on time resolution. Littlewood \& Croke (2008) show that the calibrated parameters of a unit hydrograph-based model change substantially over a range of time steps from 1 to 24 hours used in calibration and highlight the importance of accounting for model parameter data time step dependencies in pursuit of a reduction in the uncertainty of simulations. The authors also suggest that further work along these lines be undertaken using different catchments and models.

Kavetski et al. (20II) present quantitative and qualitative insights into the time scale dependencies of hydrological parameters, predictions and their uncertainties and examine the impact of the time resolution of the calibration data on model complexity. The authors argue that the use of likelihood functions that better represent the statistical description of the observed data can help in reducing time scale dependencies of model parameters and in improving the identifiability of increasingly complex model structures. They concluded that fixed-step explicit time stepping, which is usually adopted in conceptual hydrological models, can create susbtantial time scale trend in the model results. Clark \& Kavetski (2010) show that, over vast regions of the parameter space, the numerical errors of fixed-step explicit schemes commonly used in hydrology routinely dwarf the structural errors of the model conceptualization.

In the present context where rapid developments in computational processing have allowed the possibility of the estimation of flood frequency characteristics by continuous simulation, a hydrological simulation using shorter time steps is desirable as such simulations could provide better performance in short-term river discharge estimation (e.g., Wang et al. 2009). However, rainfall and runoff data with high temporal resolution are usually not available as desired. Therefore, rainfall-runoff models are commonly calibrated at coarser time scales (e.g., daily). Subsequently, parameters are then used in conjunction with finer scale input data observed or generated from stochastic rainfall generators to produce simulations with a sub-daily time step (e.g., Blazkova \& Beven 2002; Cameron et al. 2000).

In this study, we revisit the issue of temporal sampling in hydrological modelling. It is worth noting that issues related to the numerical implementation of hydrological models is not dealt with explicitly here. The conceptual model is solved using fixed-step explicit time stepping. Here we focus on deriving functional relationships between the parameters of the conceptual hydrological model HYMOD and the temporal resolution of the calibration data, so that more realistic modelling of hydrological processes can be conducted at sub-daily time steps where data availability does not allow calibration at time steps shorter than daily.

\section{METHODS}

In order to investigate the effect of temporal sampling of data on model performance and the value of derived model parameters, a numerical experiment is devised where a rainfall-runoff model is calibrated using varying resolutions of rainfall and runoff data. Subsequently, different parameter sets or 'simulators' are identified for each resolution using the generalized likelihood uncertainty estimation (GLUE) method. A functional relationship between model parameters and the data resolution is derived which is then evaluated using a period and catchment other than that used for calibration.

\section{Study area, model and data}

The method is applied to two study catchments located within the Republic of Ireland: the river Moy at Rahans $\left(1,803 \mathrm{~km}^{2}\right)$, located on the western coast of Ireland and the Boyne at Slane Castle $\left(2,452 \mathrm{~km}^{2}\right)$ located on the east coast. In the context of Irish climate, the Moy basin is wetter (SAAR $=1,323 \mathrm{~mm}$ ) and has a higher runoff coefficient than the Boyne $(\mathrm{SARR}=890 \mathrm{~mm})$. The Boyne can be considered to represent a relatively dry basin in the Irish context, whereas the Moy can be considered to represent a wet basin. Observed stream flow data were 
obtained from the Office of Public Works (available at http://www.opw.ie/hydro/), and observed precipitation and temperature data were obtained from Met Éireann, the Irish National Meteorological Service for the period 1971-2000.

To evaluate the sensitivity of model parameters to the time step used for calibration we employ the HYMOD model (see Boyle 200I; Wagener et al. 200I). HYMOD is a lumped model and is frequently used for prediction and scientific evaluation purposes. The model uses a non-linear tank connected with two series of linear tanks in parallel to model the rainfall excess mechanism. Runoff generation consists of two components, a slow flow component and a quick flow component. The model has a non-linear component which partitions precipitation into precipitation excess. Furthermore, the model has a linear routing component (Figure 1). In HYMOD, the spatial variation in store capacity over a basin is represented by the reflected power distribution function (Equation (1)).

$F(C)=1-\left(1-\frac{C}{C_{\max }}\right)^{B_{\exp }} \quad 0 \leq C \leq C_{\max }$

where $F($.$) is a water storage capacity distribution function,$ $C_{\max }$ is the parameter that defines the size of the largest water storage capacity within the catchment, $B_{\exp }$ is the parameter that defines the degree of spatial variability in the water storage capacities. The model uses a parameter, $\alpha$, to partition the water from surface storage to slow and fast reservoirs or tanks. These fast and slow reservoirs are used in the model for the translation of water to the basin outlet. For translation, the model uses a single linear reservoir to simulate the slow flow component of basins and three identical reservoirs, characterized with only a single parameter, in series to simulate the quick component. The time constant for these reservoirs are characterized by parameters $K_{\mathrm{s}}$ and $K_{\mathrm{q}}$, respectively for the slow and quick flow tanks. The model output is the summation of the flow from these two tanks. Evapotranspiration occurs at the potential rate if sufficient soil moisture is available and then occurs at a reduced level depending upon the available soil-moisture content. The slow flow component, modelled using one linear reservoir, represents the groundwater, while the quick flow component, which represents the surface flow, is modelled using three identical quick flow reservoirs. In the present application of HYMOD, a total of five parameters require estimation through model calibration. The prior uncertainty range of parameter values used for simulation is as shown in Table 1 (see also Vrugt et al. 2003).

\section{Parameter scaling}

The application of the model with coarse scale input data (e.g., daily) for modelling hydrological response at shorter time steps (e.g., sub-daily) can be achieved by suitably

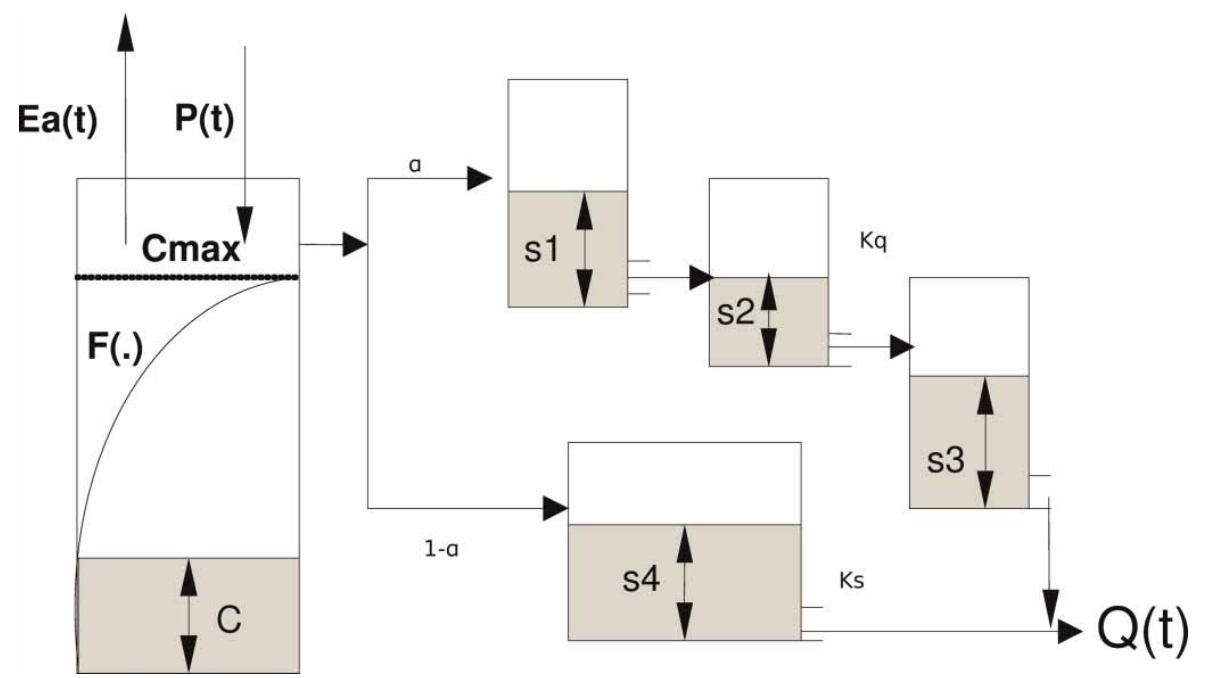

Figure 1 | Structure of HYMOD model. 


\begin{tabular}{|c|c|c|c|c|}
\hline Sn & $\begin{array}{l}\text { Description of model } \\
\text { parameter }\end{array}$ & Abbreviation & Minimum & Maximum \\
\hline 1 & $\begin{array}{l}\text { Maximum storage capacity } \\
\text { in the basin }\end{array}$ & $C_{\max }(\mathrm{L})$ & 200 & 500 \\
\hline 2 & $\begin{array}{l}\text { Spatial variability of soil- } \\
\text { moisture distribution } \\
\text { within the basin }\end{array}$ & $B_{\exp }$ & 0.1 & 2 \\
\hline 3 & $\begin{array}{l}\text { Flow distribution between } \\
\text { the quick and slow } \\
\text { linear reservoirs }\end{array}$ & $A$ & 0.2 & 1 \\
\hline 4 & $\begin{array}{l}\text { Residence time of the } \\
\text { quick release reservoir }\end{array}$ & $K_{\mathrm{q}}\left(\mathrm{day}^{-1}\right)$ & 0 & 0.9 \\
\hline 5 & $\begin{array}{l}\text { Residence time of the slow } \\
\text { release reservoir }\end{array}$ & $K_{\mathrm{s}}\left(\mathrm{day}^{-1}\right)$ & 0 & 0.1 \\
\hline
\end{tabular}

adjusting the parameters to account for the effect of the temporal sampling of data on model parameter estimation. Defining a relationship between model parameters and the temporal scale is the simplest way to make an adjustment. Here we use the equation:

$\theta_{T^{\prime}, i}^{\prime}=\theta_{T, i}+\beta_{i} \theta_{T, i}\left(T-T^{\prime}\right) / 100$

where $\theta_{T, i}$ is the $i$ th parameter estimated with the modelling time step $T$. The primed quantity refers to the desired short time step (sub-daily) and unprimed refers to longer scale time step (daily). This relationship linearly scales the value of model parameters based on desired sub-daily time step and daily time step. The scaling factor $\beta$ is estimated from the calibration dataset.

Experience with hydrological modelling and parameter calibration has shown that a large number of plausible values exist that result in behavioural parameter sets that can produce acceptable simulations from wide ranges of parameter space. One way to avoid the effect of parameter uncertainty in deriving the scaling relationship is to base the derived relationship on the median value of behavioural model parameters. The scaling relationship between the median parameter value and the time scale can be based on parameter values estimated for each 3-, 6-, 12- and 24 hour modelling time step.

Alternatively, the scaling relationship can also be obtained by simultaneously maximizing the performance for each time step with respect to chosen objective criteria.
This allows simulataneous estimation of model parameter and scaling relationships. In relation to the latter, following Bastola et al. (2008) we used the Nash-Sutcliffe efficiency (NSE) of simulated streamflow and the NSE for transformed flow, to consider the heteroscedastic variance in flow, where the flow was transformed explicitly before evaluating the objective function by using Equation (3):

$z=\left[(y+1)^{\lambda}-1\right] / \lambda$

where $\lambda$ is the transformation parameter, selected to be $0.3, z$ is the transformed streamflow, and $y$ is observed streamflow. It is referred to as HMLE (Heteroscedastic Maximum Likelihood Estimator) hereafter. For the simultaneous estimation of the model parameter and scaling relationship, a multiobjective multi-timestep calibration method is used. Multitime step calibration attempts to calibrate the model for all time steps simultaneously while concurrently attempting to achieve the best possible scaling relationship (Equation (2)) between model parameters and model time step. In this method, first the approximate functional relationship between MPs $(\theta)$ and modelling time step $(T)$ is assumed a priori (e.g., Equation (2)). Subsequently, the parameters of the functional relationship (i.e., $\alpha$ ) are calibrated such that the average of the model performance for all time steps considered for multi-time calibration is maximized. The Paretobased multi-objective approach was adopted (considering various objective criteria as mentioned above) for the calibration. In the context of multi-objective calibration, the optimization problem for the calibration of a multi-step model can be stated as follows:

$\operatorname{Maximize} F(\theta)=\left\{\left[\frac{1}{n} \sum_{i=1}^{n} f_{1, i}(\theta), \frac{1}{n} \sum_{i=1}^{n} f_{q, i}(\theta)\right]\right\}$

where $\theta$ is the vector of model parameters, $n$ is the number of parameters of the hydrological model, $n$ is the number of temporal resolutions used for calibration (e.g., four, i.e., 3hourly, 6-hourly, 12-hourly and 24-hourly), $q$ is the number of objective functions (eight objective functions are used in this study), and $f_{p, i}(\theta)$ is the model performances for the ith resolution measured with respect to objective criteria. To calibrate the regional relationship between MPs and time step, we employ a non-dominating sorting genetic 
algorithm (NSGAII) (Dev et al. 2002) which is a populationbased heuristic search algorithm which handles multiple algorithms using a Pareto-based approach. In this approach, the simultaneous maximization of performance is sought for each time step and objective criteria. The solution to Equation (4) results in a set of Pareto optimal solutions, but the compromised solution, which represents the point that is closest to the ideal performance point is chosen. For the numerical experiment, the underlying hourly precipitation and runoff measurements were averaged for increasingly longer time intervals. Subsequently, the relationships between time steps and selected sensitive parameters are calibrated.

\section{RESULTS}

\section{Sensitivity of model parameters}

The sensitivity of model parameters is tested using regional sensitivity analysis (RSA). RSA is a widely used global sensitivity method that utilizes the results of Monte-Carlo sampling (see Spear \& Hornberger 1980) to identify sensitive model parameters. In this method, each parameter's population is split into two groups of equal size and the cumulative distribution of the parameters in each group is plotted with respect to the chosen measure of performance. Differences in form and separation of the resulting curves indicate parameter sensitivity. The cumulative distributions of both groups for two different time resolutions (3-hourly and daily) for the Boyne and Moy river catchments are shown in Figure 2. For the three parameters $\alpha, K_{\mathrm{s}}$ and $K_{\mathrm{q}}$ the discrepancies between the cumulative plot corresponding to behavioural and non-behavioural populations are markedly high, a clear signature of high sensitivity. In addition, a sensitivity index, defined as the ratio of the maximum distance between the cumulative curve corresponding to behavioural and non-behavioural simulators and the median value of the corresponding calibrated model parameters is estimated to summarize the sensitivity of parameters and their dependence on time step. This index for the 3-hourly and daily time steps is shown in Figure 3. The three parameters mentioned above showed higher sensitivity. Additionally, the sensitiviy of these parameters increased when the calibration time step is decreased from daily to 3-hourly.

\section{Data resolution and model performance}

The models (or basin simulators) identified with both 3hourly and daily time steps are used to assess the impact of modelling time step on model parameters and simulation performance. The performance of behavioural simulators is assessed both in terms of timing (using NSE) and the volume of flow (using volume error). Results for the two selected catchments are shown in Figure 4. The performance criteria for the 3-hourly time step are calculated based on the simulated flow aggregated to a daily time step. Although the number of behavioural simulators are significantly less for 3-hourly simulations as compared to daily data, the spread of points in parameter space is similar.

The Moy basin shows a larger volume bias in comparison to the Boyne. Model performance is conditional upon a number of factors including the chosen objective function, the method of spatial aggregation of rainfall and the period used for calibration. In this study, as the modelling was conducted at a sub-daily time scale, only 3 years of data were used. In addition, the NSE criteria were used to estimate behavioural simulators. As there was only a single rainfall station measuring sub-hourly rainfall, we used the data from this station to represent catchment rainfall. Despite having high volume bias, the model for Moy was accepted based on NSE criteria for this study. Importantly, of primary interest to this numerical experiment are the relative differences in performance with varying modelling time steps.

When the behavioural simulators identified from one time step are used to simulate models with the other time step there is a notable shift of points in the model performance space. Figure 5 shows the impact of using daily basin simulators in the performance of model simuation with time steps shorter than that used for calibration, while Figure 6 shows the impact of using hourly basin simulators on time steps longer than that used for calibration. Figures 5 and 6 show clearly the impact for the Boyne and Moy catchments, revealing that points in model performance space moved away from the ideal performance point 
(a)
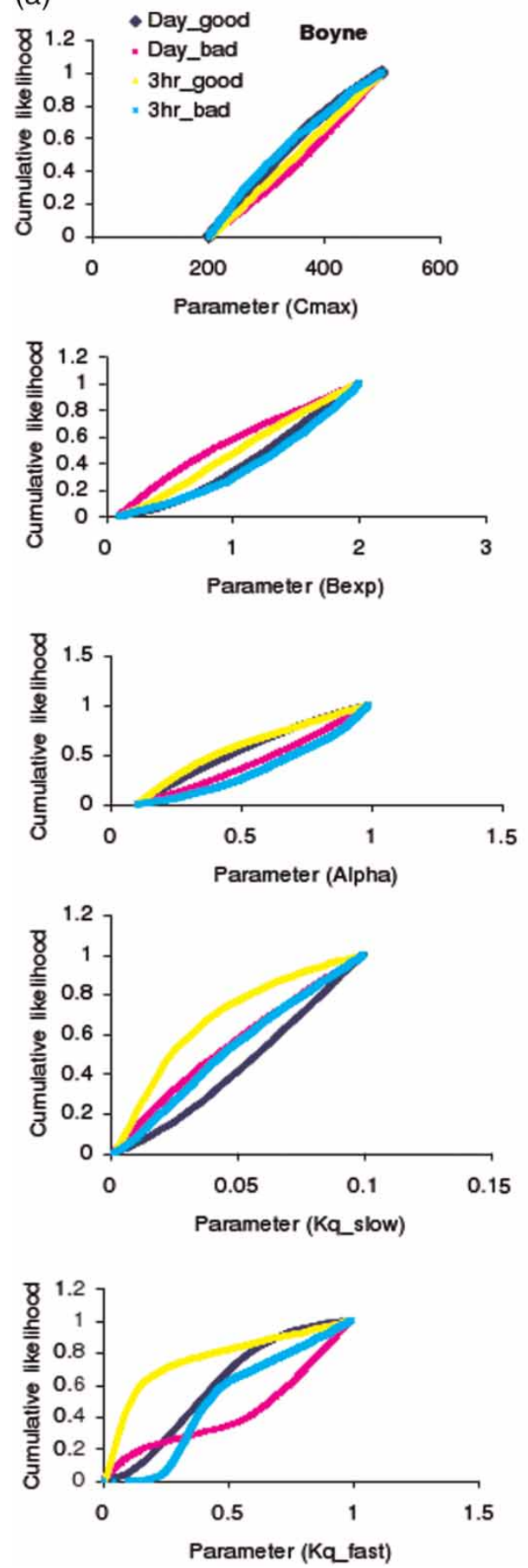

(b)
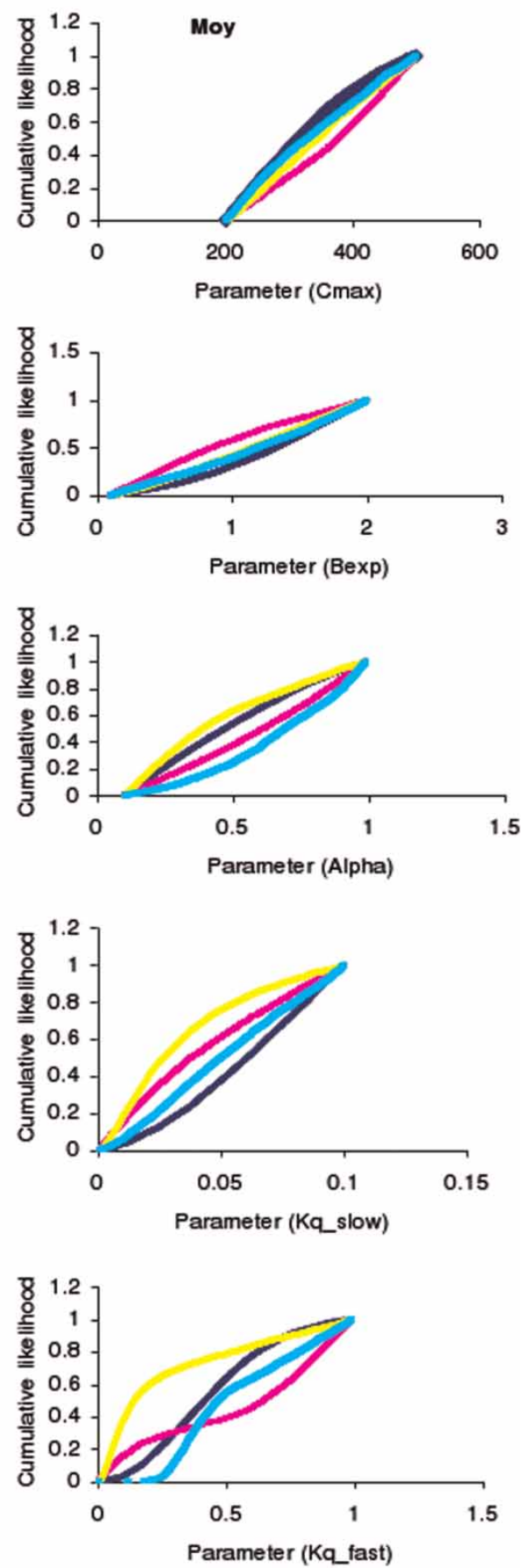

Figure 2 | Regional sensitivity analysis plots showing the sensitivity of the parameters of the HYMOD model and its sensitivity to modelling time step for (a) Boyne river catchment and (b) Moy river catchment.

$(\mathrm{NSE}=1$ and volume error $=0)$ when models identified with daily data are used for 3-hourly, 6-hourly and 12hourly simulations.
For the basins selected in this study, the loss in performance in multi-time step simulations associated with simulators calibrated with daily data is larger than that 
(a)



口Daily

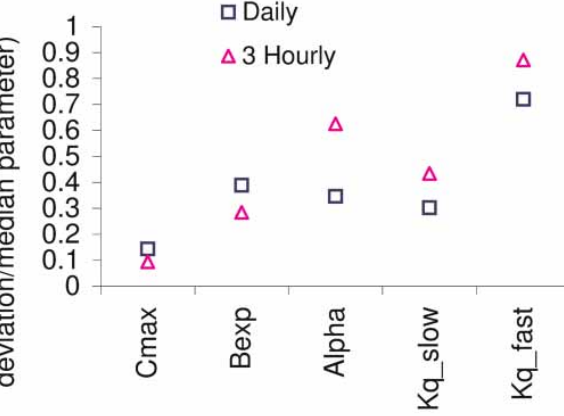

(b)

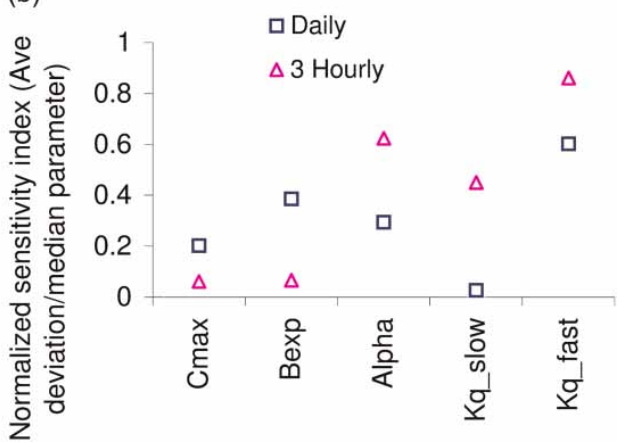

Figure 3 | Sensitivity index for the parameters of the HYMOD model for two modelling time steps: (a) Boyne river catchment; (b) Moy river catchment.

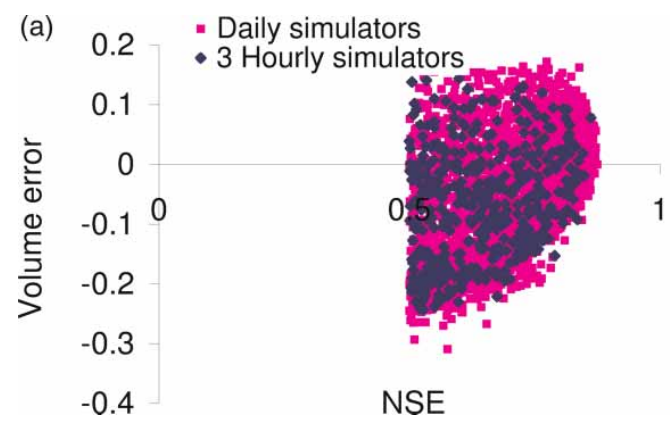

(b)



Figure 4 | Model objective space for the simulations obtained through daily and 3-hourly data for (a) Boyne river catchment and (b) Moy river catchment.



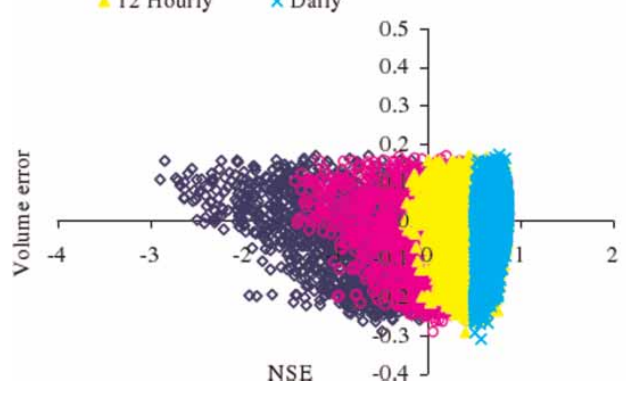

(b)



Figure 5 | Model performances of daily simulators with 3-hourly, 6-hourly, 12-hourly and daily time steps: (a) Boyne river catchment; (b) Moy river catchment. The NSE and volume error are evaluated after aggregating corresponding data to a daily time scale.

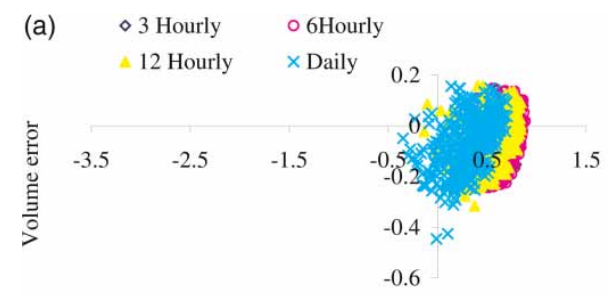

NSE (b)
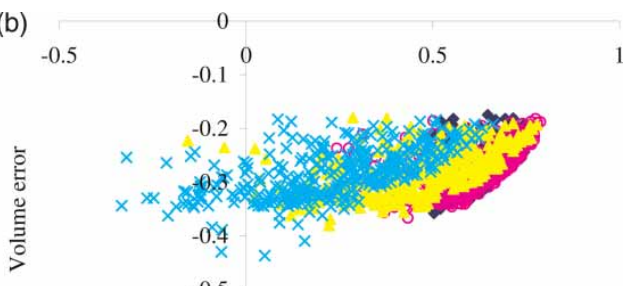

NSE

Figure 6 | Model performances of hourly simulators with 3-hourly, 6-hourly, 12-hourly and daily time steps: (a) Boyne river catchment; (b) Moy river catchment. The NSE and volume error are evaluated after aggregating corresponding data to a daily time scale. 
associated with hourly data. This supports the argument that scaling of model parameters is desirable if the simulation time step is different from the time step that is used for model calibration. Table 2 summarizes the simulation results for the Boyne catchment for the calibration and validation periods. The performance measured in terms of NSE for simulation with 3-, 6-, 12-hourly and daily time steps are acceptable. The width and reliability of the prediction interval (i.e., $\mathrm{CN}$ is the count efficiency that measures the percentage of observation encapsulated within the predicted range) evaluated using the GLUE method are also similar for all simulations made during the calibration period. However, a loss in model performance, precision (range) and reliability (count efficiency) is observed when daily simulators are used for sub-daily time step simulation and vice versa.

Figure $7(\mathrm{a})$ and $7(\mathrm{~b})$ shows the hydrograph for calibration and validation periods (3 years). It is apparent from the figure that the peaks estimated with a 3-hourly time step are greater than those estimated with a daily time step. The same is observed in the validation period. Figure 7(c) shows for the validation period the flow simulated with daily simulators but at 3-hourly and daily time steps. It is also apparent that the simulators derived from 3-hourly data tend to overestimate peak flow relative to simulators derived from a daily time step.

The fact that a loss in performance is observed when behavioural simulators derived from a daily time step are used to simulate at sub-daily time steps and given that the sensitivity of model parameters also change with modelling time step, recalibration is desirable if the modelling time step is changed. An alternative to this would be to scale the parameters to suit the modelling time step.

\section{Scaling the parameters of the HYMOD model}

In tackling this problem, particularly where appropriate data time steps may not be available, the scaling of the parameters enables adjustments to be made to account for changes in the temporal aggregation of data. This facilitates the use of information from daily hydrological records in rainfall-runoff models that operate on time steps much shorter than daily. Table 3 shows the percentage change in the model parameters observed when the modelling time step is changed from daily to sub-daily. Results are based on the median parameter value calculated from the behavioural set of model parameters. The percentage change for $\alpha, K_{\mathrm{q}}$ and $K_{\mathrm{s}}$ is markedly higher than for the $C_{\max }$ and $B_{\exp }$ parameters.

In addition to the modelling scales, the likelihood measures and uncertainty in model parameters influence the scaling relationship. Therefore, the estimation of a scaling relationship is treated as a multi-objective problem, where the scaling relationship between model parameters and data time steps is estimated using the NSGAII search algorithm.

Eight objective criteria, four based on NSE, i.e., each estimated based on 3-, 6-, 12- and 24-hourly simulation time steps, and the other four based on the HMLE criteria are used. From the Pareto optimal solutions, a scaling relationship corresponding to the compromised solution is selected. The scaling parameter ( $\alpha$ in Equation (2)) corresponding to the compromised solution is $-2.2,-2.3$ and -3.2 for the $\alpha, K_{\mathrm{s}}$ and $K_{\mathrm{q}}$ parameters, respectively. These parameters, which are derived based on data for the Boyne catchment were subsequently used to scale the

Table 2 | Performances for basin simulators estimated with different time resolution for the Boyne river catchment (CN is the count efficiency that measures the percentage of observations lying within the prediction range; Range is the average width of the prediction interval)

\begin{tabular}{|c|c|c|c|c|c|c|c|c|c|c|}
\hline \multirow[b]{3}{*}{ Sn } & \multirow[b]{3}{*}{ Modelling time step } & \multirow{2}{*}{\multicolumn{3}{|c|}{ Calibration }} & \multicolumn{6}{|c|}{ Validation } \\
\hline & & & & & \multicolumn{3}{|c|}{ With daily simulators } & \multicolumn{3}{|c|}{ With 3-hourly simulators } \\
\hline & & CN & NSE & Range & CN & NSE & Range & CN & NSE & Range \\
\hline 1 & 3-hourly & 0.85 & 0.78 & 25.07 & 0.46 & 0.63 & 16.07 & 0.75 & 0.75 & 24.77 \\
\hline 2 & 6-hourly & 0.86 & 0.78 & 24.42 & 0.67 & 0.71 & 21.12 & 0.75 & 0.78 & 22.99 \\
\hline 3 & 12-hourly & 0.91 & 0.81 & 24.55 & 0.73 & 0.75 & 22.91 & 0.64 & 0.71 & 19.99 \\
\hline 4 & 24-hourly & 0.92 & 0.82 & 24.53 & 0.79 & 0.79 & 24.95 & 0.44 & 0.61 & 16.63 \\
\hline
\end{tabular}



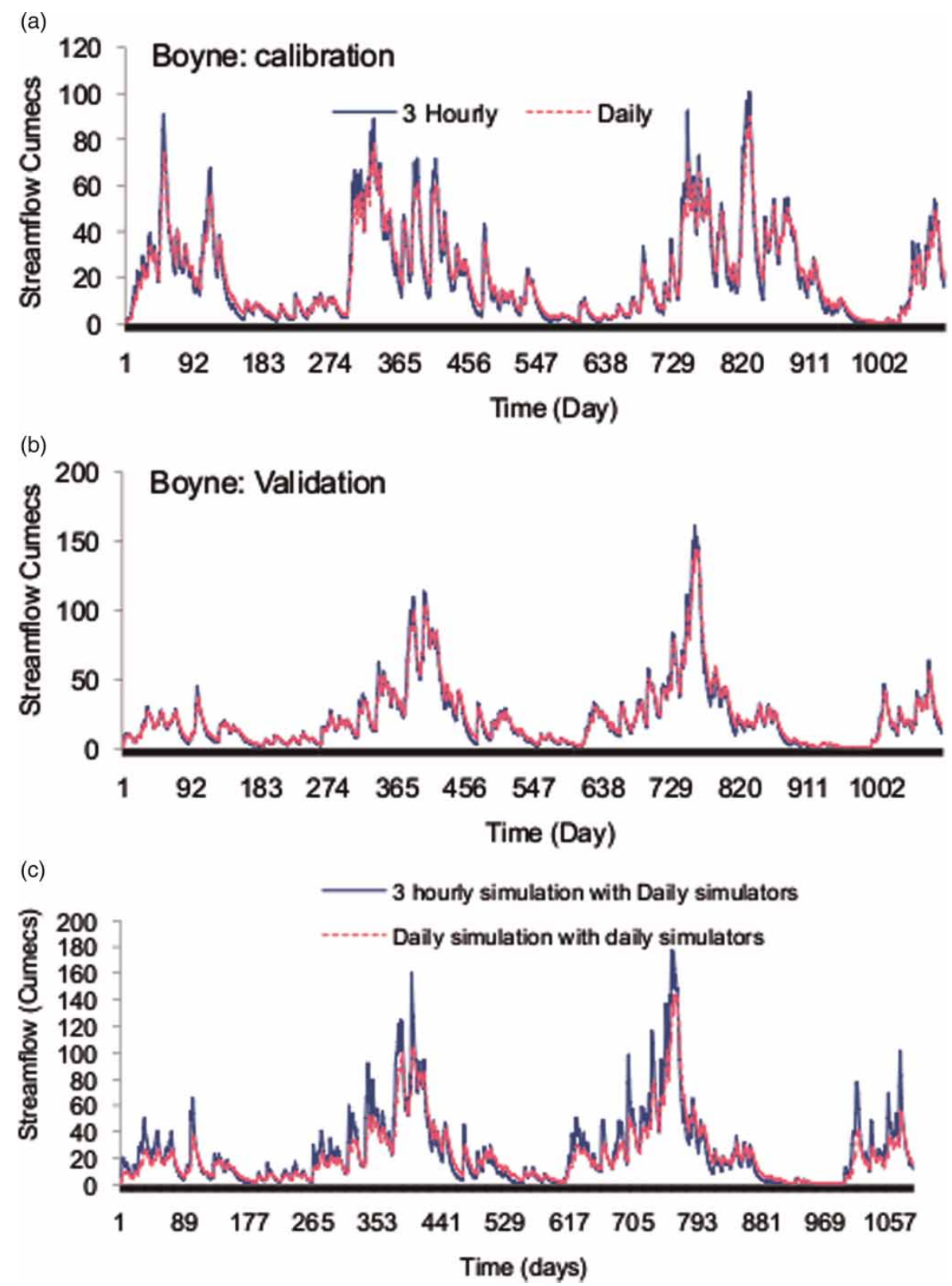

Figure 7 | Simulation results for (a) calibration (Boyne river catchment), (b) validation (Boyne river catchment), (c) validation period with scaled and unscaled simulators.

parameters for the Moy catchment. The performance of the scaled parameters for the validation period (period not used for calibration) and catchment (Moy river catchment) is shown in Figure 8. Compared to the unscaled parameters, i.e., using daily basin simulators for simulation of sub-daily time steps, the loss in model performance corresponding to the use of scaled parameters is smaller. Table 4 summarizes the simulation results for the Boyne catchment with rescaled basin simulators used for the validation period.

\section{CONCLUSION}

This study revisits the issue related to the temporal sampling of data and the performance of a hydrological model using 
Table 3 Percentage change in the value of parameters identified with daily time step simulation when the daily simulation time step is reduced to sub-daily

\begin{tabular}{|c|c|c|c|c|c|c|c|}
\hline \multirow[b]{2}{*}{ sn } & \multirow[b]{2}{*}{ Basin } & \multirow[b]{2}{*}{ Time resolution (hour) } & \multicolumn{5}{|c|}{$\%$ change in parameter with respect to daily calibrated parameter } \\
\hline & & & $c_{\max }$ & $B_{\text {exp }}$ & $\alpha$ & $\boldsymbol{\kappa}_{\mathbf{q}}$ & $\boldsymbol{\kappa}_{\mathbf{s}}$ \\
\hline 1 & Boyne & 3 & 2.1 & 6.7 & -41.6 & -73.4 & -49.3 \\
\hline 2 & & 6 & 1.9 & 3.2 & -21.3 & -37.9 & -47.0 \\
\hline 3 & & 12 & 1.1 & 1.8 & -12.3 & -9.2 & -19.0 \\
\hline 4 & Moy & 3 & -4.9 & 22.5 & -53.6 & -81.1 & -37.6 \\
\hline 5 & & 6 & 0.1 & 19.3 & -35.2 & -62.5 & -39.5 \\
\hline 6 & & 12 & -0.6 & 9.2 & -15.7 & -21.4 & -28.6 \\
\hline
\end{tabular}

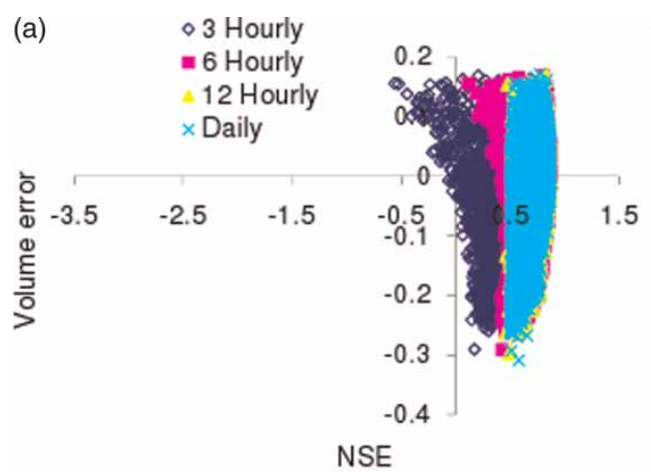

(b)

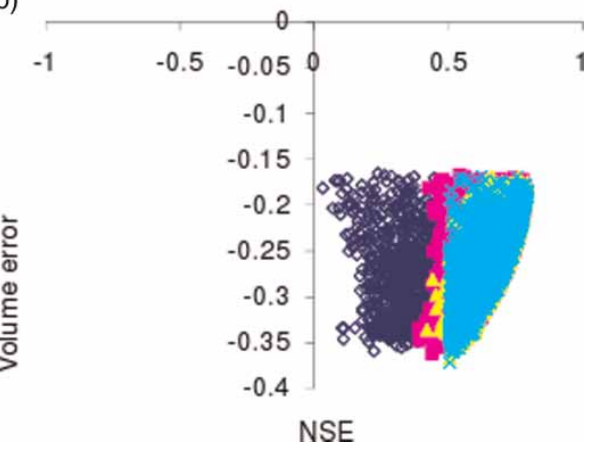

Figure 8 Model performances of scaled (from daily to sub-daily) simulators for two river catchments: (a) Boyne; (b) Moy. The NSE and volume error are evaluated after aggregating corresponding data to a daily time scale.

Table 4 | Performance for scaled basin parameters for the Boyne river catchment (CN is the count efficiency that measures the percentage of observation lying within the prediction range; Range is the average width of the prediction interval)

\begin{tabular}{lllllllll} 
& & \multicolumn{3}{l}{ Calibration } & & & \multicolumn{2}{l}{ Validation } \\
\cline { 3 - 4 } Sn & Modelling time step & CN & NSE & Range & & CN & NSE & Range \\
\hline 1 & 3-hourly & 0.83 & 0.76 & 22.16 & 0.68 & 0.71 & 21.07 \\
2 & 6-hourly & 0.87 & 0.79 & 22.71 & 0.71 & 0.75 & 21.82 \\
3 & 12-hourly & 0.91 & 0.82 & 23.47 & 0.74 & 0.78 & 22.79 \\
4 & 24-hourly & 0.92 & 0.82 & 23.90 & 0.79 & 0.79 & 24.94 \\
\hline
\end{tabular}

two Irish river catchments. A straightforward way of applying a hydrological model at different time scales is to adjust parameters depending upon the scale at which parameters are calibrated and the temporal scale at which simulation is desired. The HYMOD model is run in a continuous mode for a 3-year period for calibration and another 3-year period for validation using model time steps of 3, 6 $12 \mathrm{~h}$ and daily. Runoff timing and volume biases are investigated when performing simulations at time scales different from those at which the model parameters are calibrated. The analysis revealed a general increase in modelled peaks when moving from longer to shorter time steps.

The use of parameters estimated using daily time steps resulted in a significant loss in model performance when applied to 3-hourly time steps. However, simple adjustment of the parameters resulted in improved model simulations, reducing the need for recalibration and indicating the potential for the application of such techniques in situations where sub-daily data may not be available. The results shown indicate that it is necessary to adjust parameters derived from daily time steps if sub-daily simulations are to be made. Furthermore, the parameter adjustment procedure is evaluated by applying such procedures during periods and in catchments other than used for calibration. 


\section{ACKNOWLEDGEMENTS}

This work received financial support from Science Foundation Ireland's Research Frontiers Programme under the project title 'Quantifying the cascade of uncertainty in climate change impacts for the water sector'. We are also grateful to Peter Newport of Hydrometric section of the Office of Public Works for providing instantaneous 15 minute flow data for two stations. We thanks the anonymous reviewers for their insightful comments which have helped to improve this paper. We also acknowledge Prof John Sweeney for his continuous support and suggestions throughout the work.

\section{REFERENCES}

Bastola, S., Ishidahira, H. \& Takeuchi, K. 2008 Regionalisation of the parameters of hydrological model under parameter uncertainty: A case study involving TOPMODEL and basins across the globe. J. Hydrol. 357 (3-4), 188-206.

Bastola, S., Murphy, C. \& Sweeney, J. 2orn The role of hydrological modelling uncertainties in climate change impact assessments of Irish river catchments. Adv. Water Resour. 34 (5), 562-576.

Blazkova, S. \& Beven, K. 2002 Flood frequency estimation by continuous simulation for a catchment treated as ungauged (with uncertainty). Water Resour. Res. 38 (8), 1139.

Boyle, D. 200I Multicriteria calibration of hydrological models. PhD dissertation. Department of Hydrology and Water Resources, University of Arizona, Tucson, AZ.

Cameron, D., Beven, K., Tawn, J. \& Naden, P. 2000 Flood frequency estimation by continuous simulation (with likelihood based uncertainty estimation). Hydrol. Earth Syst. Sci. 4 (1), 23-34.

Clark, M. P. \& Kavetski, D. 20Io The ancient numerical demons of conceptual hydrological modeling: 1. Fidelity and efficiency of time stepping schemes. Water Resour. Res. 46, W10511.
Dev, K., Agrawal, S., Pratap, A. \& Meyarivan, T. 2002 A fast and elitist multi-objective Genetic Algorithm: NSGA-II. Trans. Evol. Comput. 6, 182-197.

Finnerty, B. D., Smith, B. M., Seo, D. J., Koren, V. \& Moglen, G. E. I997 Space-time scale sensitivity of the Sacramento model to radar gauge precipitation inputs. J. Hydrol. 203, 21-38.

Ishidaira, H., Takeuchi, K., Xu, Z., Ao, T., Magome, J. \& Kudo, M. 2003 Effect of spatial and temporal resolution of precipitation data on the accuracy of long-term runoff simulation. IAHS Publ. 282, 186-193.

Kavetski, D., Fenicia, F. \& Clark, M. P. 2or Impact of temporal data resolution on parameter inference and model identification in conceptual hydrological modeling: Insights from an experimental catchment. Water Resour. Res. 47, W05501.

Krajewski, W. F., Lakshmi, V., Georgakakos, K. P. \& Jai, S. C. I99I A monte carlo study of rainfall sampling effect on a distributed catchment model. Water Resour. Res. 27 (1), $119-128$.

Littlewood, I. G. \& Croke, B. F. W. 2008 Data time-step dependency of conceptual rainfall-streamflow model parameters: An empirical study with implications for regionalization. Hydrol. Sci. J. 53 (4), 685-695.

Ostrowski, M., Bach, M., DeSimone, S. V. \& Gamerith, V. 2010 Analysis of the time-step dependency of parameters in conceptual hydrologic models. TU-Darmstadt (Germany), Report, DOI/URN:urn:nbn:de:tuda-tuprints-20996.

Spear, R. C. \& Hornberger, G. M. I980 Eutrophication in Peel Inlet, II, Identification of critical uncertainties via generalized sensitivity analysis. Water Research 14, 43-49.

Vrugt, J. A., Gupta, H. V., Bastidas, L. A., Bouten, W. \& Sorooshian, S. 2003 A shuffled complex evolution metropolis algorithm for optimization and uncertainty assessment of hydrologic model parameters. Water Resour. Res. 39 (8), 1201.

Wagener, T., Boyle, D. P., Lees, M. J., Wheater, H. S., Gupta, H. V. \& Sorooshian, S. 200I A framework for development and application of hydrological models. Hydrol. Earth Sci. 5 (1), 13-26.

Wang, Y., He, B. \& Takase, K. 2009 Effects of temporal resolution on hydrological model parameters and its impact on prediction of river discharge. Hydrol. Sci. J. 54 (5), 886-898.

First received 22 March 2012; accepted in revised form 3 April 2012. Available online 22 November 2012 